\title{
3D molecular dynamic simulation of fracture behavior of bcc iron
}

\author{
Bo $\mathrm{Ma}^{1, \mathrm{a}}$ \\ ${ }^{1}$ College of Electrical \& Information Engineering, Southwest University for Nationalities, Chengdu \\ 610000,PR China \\ amabo3721@sina.com
}

Keywords: fracture; bcc iron; molecular dynamic simulation; EAM potential

\begin{abstract}
The fracture behavior of bcc iron is investigated by means of 3D molecular dynamic simulation with EAM potential. The crack propagation is studied under the condition of specific temperatures and loading. The results show that the formation of dislocation is different according to the strain rates. The temperature has a significant effect on the crack profile and dislocation. Slip on (110) plan is observed in the crack process.
\end{abstract}

\section{Introduction}

Iron is an important material for vehicle. It is a strong metal used widely with good deformation and alloying property. Understanding the process of crack and fracture of iron is essential to safe utilization of them in various structures. The basic fracture mechanics is the linear elastic fracture mechanics (LEFM), which is an ideal approach to dealing with linear elastic materials. SK Chan i.e. proposed the finite element method of linear fracture mechanics to compute crack tip stress intensity factors. Beside of continuum approaches to studding fracture, molecular dynamic method is another means of investigation of fracture at the atomic scale. Since the first molecular dynamic simulation of crack propagation was reported in 1976 by Ashurst and Hoover, [1] the fracture of iron has been studied widely by the means of MD simulation. The simulations were small (1000 10000 atoms) 2D systems at the early stage for the limitation of the computing power. In order to deal with the crack propagation in small geometry of system various boundary conditions were introduced. DeCelis, Argon, and Yip proposed a constant-stress boundary condition [2] to eliminate some of the difficulties of dealing with the transmission of the dislocation emitted from the crack. Cheung and Yip reported that dislocations are emitted form crack tip, when they used the constant-force boundary condition in their small (3000 atoms) 3D system to study the brittle to ductile transition of bcc iron. [3] Many studies with the MD approach are 2D system or small 3D system. We report our 3D simulation to investigate the crack process in a system at nanometer scale which consisted of 60080 atoms by means of parallel computing.

\section{Simulation Method}

\section{A. Potentials}

Potential is essential to the MD simulation. MD simulations of fracture behavior of metal have been reported with various potential. DeCelis, Argon, and Yip utilized the Johnson and Morse potentials to study the intrinsic crack tip processes of either propagation by cleavage or blunting by the nucleation of dislocations from the nonlinearly stressed region at the crack tip. [2] Holian and Ravelo reported on a fracture simulation of mode-I tensile loading at high strain rates utilizing LJ and EAM potential in their study. In our simulation we utilize the EAM potential which is suited for simulation of metal. [4] EAM is a semi-empirical potential, which was made by Daw and Baskes, based on the DFT theory considering that the atom energy only depends on the density function of electron. Accordingly in the original idea of EAM potential, atoms is considered to be embedded in "cloud" of electron from anther atoms. The energy of an atom is composed of tow parts. One is the embedding energy depending on the density of the electron from neighbor atoms, another is pair 
energy contributed from the interaction from neighbors. In the system the total energy Ei of an atom $i$ is given by

$$
\begin{aligned}
& E_{i}=F_{\alpha}\left(\rho_{\alpha}\right)+\frac{1}{2} \sum_{j \neq i} \varphi_{\alpha \beta}\left(r_{i j}\right) \\
& \rho_{\alpha}=\sum_{i \neq j} f\left(r_{i j}\right)
\end{aligned}
$$

where $F$ is the embedding energy which is a function of the atoms electron density $\rho, \varphi$ is the pair energy of the interaction of atom $i$ and $j, \alpha$ and $\beta$ is the element type of atom $i$ and $j$. In the equation (2), $\mathrm{f}(\mathrm{r})$ is the electron density which can be calculated approximately from Hartree-Fock method. Function $\mathrm{F}$ and $\varphi$ in equation (1) can be fitted from the experimental data. Another method of EAM known as EAM_fs was proposed by Finnis and Sinclair[], takes the same form of equation (1), except $\rho$ is the functional specific to the atom type of $i$ and $j$. In our simulation, EAM_fs is used.

B. Setup of the Simulation

The system studied is consisted of a sample of bcc iron (60080 atoms) with rectangular shape with the length: $17.16 \mathrm{~nm}$, width: $14.3 \mathrm{~nm}$, thickness: $2.86 \mathrm{~nm}$. A rectangle crack with length: $5.726 \mathrm{~nm}$, width: $0.5726 \mathrm{~nm}$ is cut initially at the middle of the left boundary of the sample. (Fig. 1)

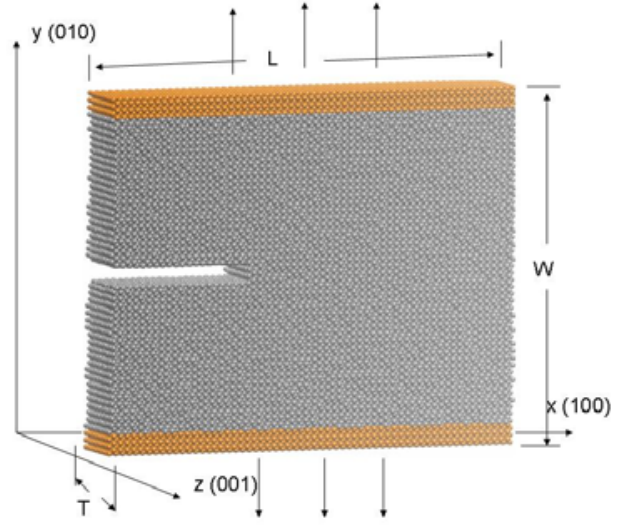

Fig. 1 The sample of bcc iron consisted of 60080 atoms.

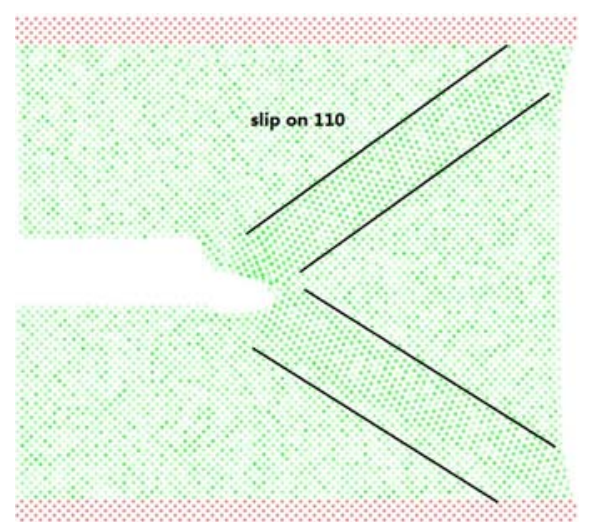

Fig.2 Slip on the 110 plane

The iron atoms of sample are classified into tow types noted boundary layer and Newton layer atom. The mode-I loading is loaded on the upper and lower boundary. By means of setting a specific velocity of the boundary atoms, the Newton layer can get corresponding strain rate. The trajectory of the atoms in Newton layer is calculated by Verlet method with EAM_fs potential. All simulation of our experiment is done by an open source program named Large scale Atomic/Molecular Massively Parallel Simulator (LAMMPS), and the result is visualized by VMD. Two steps are taken in the simulation, system relax and crack process in NVT evolution. The simulation system is set up in LAMMPS with the parameter in the Table 1. Simulation runs in 5 ps with NVT and another 5 ps with NVE to give all atoms fully relaxed .The time step of simulation is $1 \mathrm{fs}$. After the relaxation system energy reaching to a stable value that indicates the system is in the equilibrium state. (Fig.2) Two sets of simulation are performed which one is that specific loading is loaded under a constant temperature, another is that sample is under a constant loading with specific temperature . The simulation condition is given in the Table 2 . 
Table1: The parameters of the simulation

\begin{tabular}{|l|l|}
\hline sample & bcc iron \\
\hline dimension & $\begin{array}{l}17.16[\mathrm{~nm}], 14.3[\mathrm{~nm}], 2.86 \\
{[\mathrm{~nm}](\alpha=0.286[\mathrm{~nm}])}\end{array}$ \\
\hline time step & $1 \mathrm{fs}$ \\
\hline $\begin{array}{l}\text { load } \\
\text { direction }\end{array}$ & $(010)$ \\
\hline boundary & $\begin{array}{l}\text { x: shrink wrapping, } \\
\text { y: shrink wrapping, } \\
\text { z: periodic }\end{array}$ \\
\hline
\end{tabular}

Table 2: Condition of the simulation

\begin{tabular}{|l|c|c|}
\hline No & temperature[k] & velocity $[\mathrm{m} / \mathrm{s}]$ \\
\hline 1 & 300 & 10 \\
\hline 2 & 300 & 20 \\
\hline 3 & 300 & 30 \\
\hline 4 & 300 & 40 \\
\hline 5 & 300 & 50 \\
\hline 6 & 30 & 20 \\
\hline 7 & 150 & 20 \\
\hline
\end{tabular}

\section{Result}

In our simulation, center symmetry parameter (CSP) is used to investigate the disorder of the local lattice. CSP is defined as

$$
C S P=\sum_{i=1}^{4}\left|\vec{R}_{i}+\vec{R}_{i+4}\right|^{2}
$$

For an atom on a lattice site, if the neighbor atoms is on the perfect lattice, the CSP of the atom will be 0 . If the symmetry is broken, the CSP will be a larger positive value. More disorder, larger the value is .The simulation runs in $80 \mathrm{ps}$ at the temperature of $300 \mathrm{~K}$, under the strain rate caused by setting the velocity of boundary atoms at $10 \mathrm{~m} / \mathrm{s}, 20 \mathrm{~m} / \mathrm{s}, 30 \mathrm{~m} / \mathrm{s}, 40 \mathrm{~m} / \mathrm{s}$ and $50 \mathrm{~m} / \mathrm{s}$. We find that a larger strain rate can cause a larger CSP which means the system becomes more disordered. (Fig.4)

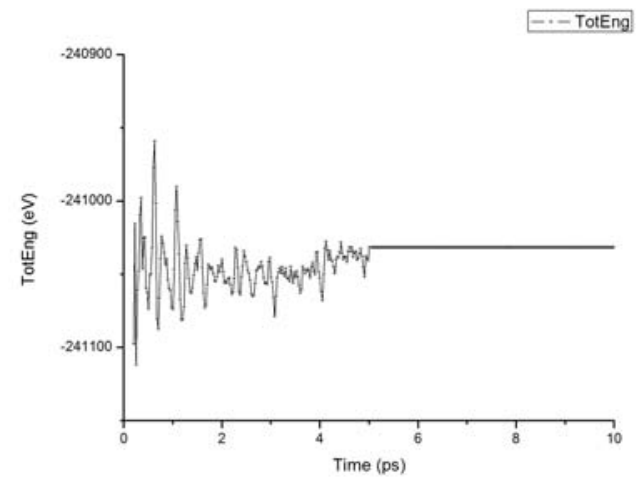

Fig.3 The total energy of system

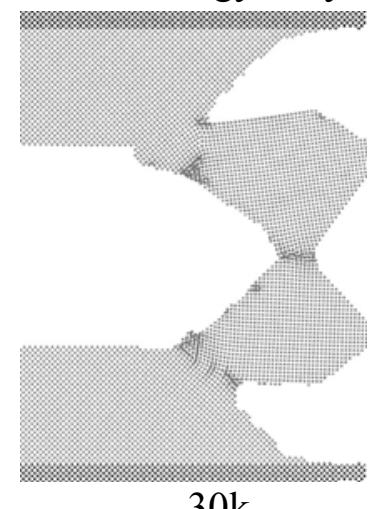

$30 \mathrm{k}$

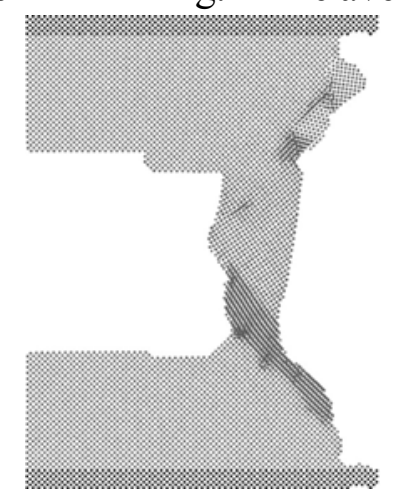

$150 \mathrm{k}$

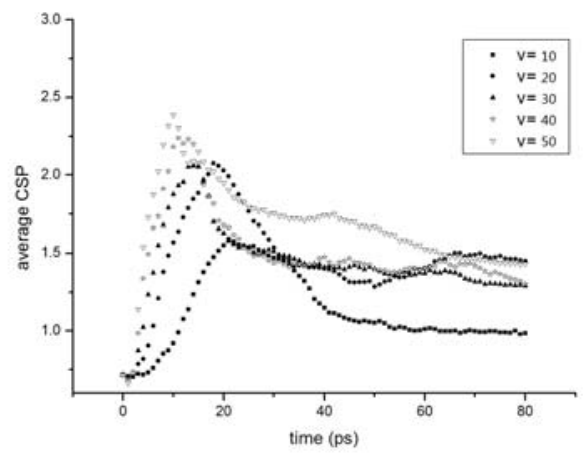

Fig.4 The average of the CSP at specific strain rate

Fig. 5 The crack at specific temperature

Fig. 5 shows the crack view at the temperature of $30 \mathrm{k}, 150 \mathrm{k}, 300 \mathrm{k}$, under the velocity of $20 \mathrm{~m} / \mathrm{s}$. It is observed that the slip on the (110) plane is significant at $300 \mathrm{k}$. 
In the crack growth process, we find that the CSP of atoms in the (110) direction is significant larger than others. In Fig. 2 the CSP of atoms is virtualized with color scale, which lighter color means smaller CSP value, darker means larger.

\section{References}

[1] W. T. Ashurst and W. G. Hoover, Phys. Rev. B 14(1976), p.1465

[2] B. deCelis, A. S. Argon, and S. Yip, J. Appl. Phys. 54 (1983), p. 4864

[3] K. S. Cheung and S. Yip, Phys. Rev. Lett. 65(1990) p.1804

[4] B. L. Holian and R. Ravelo, Phys. Rev. B 51(1995) p. 11275

[5] Information http://lammps.sandia.gov 